\title{
Identification of a potent epigenetic biomarker for resistance to camptothecin and poor outcome to irinotecan-based chemotherapy in colon cancer
}

\author{
YUICHIRO MIYAKI $^{1}$, KOICHI SUZUKI ${ }^{2}$, KEI KOIZUMI $^{1}$, TAKAHARU KATO ${ }^{2}$, MASAAKI SAITO ${ }^{2}$, \\ HIDENORI KAMIYAMA ${ }^{2}$, TAKAFUMI MAEDA ${ }^{2}$, KIYOSHI SHIBATA ${ }^{3}$, NORIHIKO SHIYA ${ }^{1}$ and FUMIO KONISHI ${ }^{2}$ \\ ${ }^{1}$ First Department of Surgery, Hamamatsu University School of Medicine, 1-20-1 Handayama, Higashi-ku, Hamamatsu, \\ Shizuoka 431-3192; ${ }^{2}$ Department of Surgery, Saitama Medical Center, Jichi Medical University, 1-847, Amanuma-cho, \\ Omiya-ku, Saitama, Saitama 330-8503; ${ }^{3}$ Research Equipment Center of Hamamatsu University School of Medicine, \\ 1-20-1 Handayama, Higashiku, Hamamatsu, Shizuoka 431-3192, Japan
}

Received July 13, 2011; Accepted August 23, 2011

DOI: 10.3892/ijo.2011.1189

\begin{abstract}
Drug resistance remains a major obstacle to successful cancer treatment. Genome-wide comprehensive analysis identified a novel gene, glucocorticoid-induced protein-coding gene (DEXI), which was frequently methylated in colorectal (CRC; 36 of 73 patients; $49 \%$ ) and gastric (28 of 89 patients; $31 \%$ ) cancer patients. Here, we show that DEXI methylation is implicated in mechanisms facilitating resistance to camptothecin (CPT) via inhibition of apoptosis. Silencing of DEXI by siRNA significantly reduced CPT-induced apoptosis in a fibroblast cell line (1/6-fold; $\mathrm{p}<0.01)$ originally expressing endogenous DEXI. Restored expression of DEXI by 5-aza-2'-deoxycytidine (DAC) significantly enhanced susceptibility to CPT ( 3 -fold; $p<0.01$ ) in a colon cancer cell line originally suppressing endogenous DEXI due to almost complete methylation. Exogenous induction of DEXI confirmed that DEXI per se contributed to enhanced susceptibility to CPT. 5-Fluorouracil (5-FU) did not exhibit these synergistic effects by DEXI restoration. Further, to estimate the clinical usefulness of DEXI methylation status as biomarker for drug resistance to irinotecan (CPT-11), 16 CRC patients who underwent FOLFIRI (5-FU + CPT-11) therapy because they were refractory to FOLFOX (5-FU + oxaliplatin) were analyzed. Significantly poor response and outcome were observed in 8 CRC patients harboring DEXI methylation. In 8 CRC patients harboring DEXI methylation disease control rate, progression-free survival and overall survival were $25.0 \%, 2$ and 11.8 months, respectively, whereas in 8 CRC patients without DEXI methylation they were $62.5 \%, 5.3$ and 15
\end{abstract}

Correspondence to: Dr Koichi Suzuki, Department of Surgery, Saitama Medical Center, Jichi Medical University, 1-847 Amanuma-cho, Omiya-ku, Saitama, Saitama 330-8503, Japan

E-mail: ksuzbnhm@yahoo.co.jp

Key words: colorectal cancer, drug resistance, apoptosis, promoter methylation, glucocorticoid-induced protein-coding gene months, respectively $(\mathrm{p}<0.01)$. These significant differences were not observed in patients undergoing treatment with FOLFOX. In conclusion, silencing of DEXI leads to resistance, but restored expression enhances susceptibility to CPT in vitro and DEXI methylation results in poor response and outcome to CPT-11-based chemotherapy, suggesting that DEXI is a potent therapeutic target and an epigenetic biomarker for the selection of patients more likely to benefit from CPT-11-based chemotherapy.

\section{Introduction}

Metastatic colorectal cancer (CRC) varies greatly in clinical outcome, depending on the response to chemotherapies despite recent advances achieved by combining new anticancer agents. One of the key drugs for metastatic CRC is irinotecan (CPT11), a derivative drug of camptothecin (CPT) (1-4) and potent DNA topoisomerase I (top I) inhibitor that blocks the rejoining step of the cleavage/religation reaction of top I (5). CPT-11 is indispensable to chemotherapy in various cancer treatments and is widely used in combination treatment using 5 -fluorouracil (5-FU) and CPT-11 (FOLFIRI) (5), which yields a high response rate $(>50 \%)$ in first-line treatment for metastatic CRC patients (6). However, progression-free survival (PFS) was estimated to be only 8-9 months when this regimen was given as first-line therapy (6), suggesting that most patients acquired resistance to CPT-11 within $<1$ year after initial treatment.

Sequential treatments using several key drugs such as $5-\mathrm{FU}$, CPT-11 and oxaliplatin (L-OHP), FOLFOX (5-FU + L-OHP) followed by FOLFIRI, or FOLFIRI followed by FOLFOX contributed to survival improvement; however, response rate to FOLFIRI or FOLFOX as second-line therapy was reported to account for only 4 and $15 \%$, respectively, suggesting that second-line therapy has limited benefit in a minority of patients regardless of treatment sequence (6). Hence adequate novel strategies are urgently required to circumvent drug resistance, which remains a major obstacle to successful cancer treatment.

Several hypotheses have been proposed for the mechanisms of resistance to CPT, including reduced cellular accumulation 
of CPT, alteration in the structure or location of top I, alterations in the cellular response to CPT-DNA-ternary complex formation (7), and activation of transcriptional factor $\kappa \mathrm{B}(7,8)$. Although multiple experimental approaches have been tried clinically for their potential ability to overcome individual mechanisms for this resistance (7), they are still far from successful. Recently, much emphasis is being placed on the critical role of epigenetic alterations in cancer progression. During CRC progression, aberrant methylation alterations appear to be accumulated such as selective hypermethylation of tumor suppressor, cell cycle regulator and proapoptotic genes (9). Epigenetic silencing of proapoptotic genes suggests that epigenetic modifications are likely involved in the machinery underlying drug resistance.

Methylation-sensitive amplified fragment-length polymorphism (MS-AFLP) is a fingerprinting technique to analyze simultaneously DNA methylation in hundreds of loci (10). This technique is based on digestion of genomic DNA with the methylation-sensitive restriction enzyme NotI and methylation insensitive restriction enzyme MseI followed by ligation of specific adaptors and subsequent PCR amplification of the generated restriction fragments. This technique provides an unbiased view of the complex picture of hypermethylation and hypomethylation, which enabled us to demonstrate that age-dependent accumulation of DNA demethylation preceded diploidy loss in a subset of gastrointestinal cancers (11). Using this technique, we identified a novel gene, glucocorticoid-induced protein-coding gene (DEXI), which was frequently methylated in our panel of colorectal and gastric cancer patients.

DEXI was first reported as a gene upregulated by dexamethasone in emphysema (12). Analysis of the protein sequence suggests that the protein has a predicted central transmembrane domain and a carboxy-terminal leucine zipper. The leucine zipper motif is present in many transcription factors and gene regulatory proteins (13). The carboxy-terminal leucine zipper contains a predicted casein kinase II phosphorylation site, suggesting that the DEXI interactions, either with itself, by dimerization, or another protein, may be regulated by phosphorylation (12). Only slight similarity is known to any other genes and no definite function has been reported. In this study, we show that DEXI methylation is implicated in mechanisms facilitating resistance to $\mathrm{CPT}$ via inhibition of apoptosis. In addition, we demonstrate clinical usefulness of DEXI methylation status as a potent epigenetic biomarker for selection of patients more likely to benefit from CPT-11-based chemotherapy.

\section{Patients and methods}

Patients and tissues. Seventy-three primary CRC and 89 primary gastric cancers with matched normal tissues from patients who had undergone surgical operations were collected as freshfrozen tissues from the Cooperative Human Tissue Network (USA) and National Cancer Center Hospital (Tokyo, Japan), respectively. Thirty-six patients who underwent FOLFOX therapy in Saitama Medical Center, Jichi Medical University were recruited and their cancer tissues collected as freshfrozen tissues as well as 12 normal healthy colon epithelia. Among them, 16 patients underwent FOLFIRI because they were refractory to FOLFOX. This study was approved by the Ethics Committee of Jichi Medical University; informed consent was obtained from each participant.

Cell lines and cell cultures. Nine different cell lines including 4 gastric cancer cell lines (H111, GSS, HGC27, AZ521), 4 CRC cell lines (CW2, CACO2, HCT116, COLO320DM), and a normal fibroblast cell line (NHDF) were used. H111, GSS, HGC27, AZ521, CW-2 and CACO2 were provided by the RIKEN Cell Bank (RCB, Tsukuba, Japan). HCT116 was obtained from the American Type Culture Collection (ATCC, Manassas, VA, USA). COLO320DM was obtained from the Japanese Collection of Research Bioresources (JCRB, Osaka, Japan). NHDF was obtained from Kurabo (Osaka, Japan). These cell lines were cultured according to the manufacturer's instructions.

DNA and RNA extraction. DNA was extracted by DNeasy ${ }^{\circledR}$ blood and tissue kit (Qiagen, Hilden, Germany). Total RNA was extracted from tissue culture cell lines by TRIzol ${ }^{\circledR}$ Plus RNA purification kit (Invitrogen, Carlsbad, CA, USA).

Methylation-sensitive amplified fragment-length polymorphism. MS-AFLP was performed as described previously (11). The primer sequence for NotI primer was 5'-GACTGCGT AGGGGCCGCG-3'. Primer sequences for 3 different MseI primers were 5'-GATGAGTCCTGAGTAAC-3' (MseI-C), 5'-GATGAGTCCTGAGTAACA-3' (MseI-CA), and 5'-GATG AGTCCTGAGTAACG-3' (MseI-CG). Each PCR sample was electrophoresed on a denaturing gel (Sequagel-6; National Diagnostics, Atlanta, GA, USA) after heat denaturing. The gel was dried and exposed to X-ray film. Scoring of quantitative changes between normal and tumor DNA was made by visual inspection as described previously (11).

Restriction enzyme-based methylation-specific PCR analysis. Genomic DNA was digested with restriction emzyme NotI (methylation sensitive) and MseI (non-sensitive for methylation). Then, PCR was performed using a set of primers to sandwich NotI restriction site located in the target gene, DEXI. PCR amplification was carried out in a $20-\mu 1$ reaction mixture containing 50 ng DNA templates, $1 \mathrm{X}$ PCR buffer, $0.25 \mathrm{mM}$ dNTP mix, $0.025 \mathrm{U} / \mu 1$ HotStarTaq Plus DNA Polymerase (Qiagen), and $0.5 \mu \mathrm{M}$ of each primer. Primer sequences were 5'-CAGAGGGTATCAACGCAATG-3' and 5'-GCGGCTC AGTGTCTAGGG-3'. PCR started at $95^{\circ} \mathrm{C}$ for $5 \mathrm{~min}$ and $95^{\circ} \mathrm{C}$ for $45 \mathrm{sec}$ followed by 35 cycles of $95^{\circ} \mathrm{C}$ for $45 \mathrm{sec}, 56^{\circ} \mathrm{C}$ for $45 \mathrm{sec}$, and $72^{\circ} \mathrm{C}$ for $1 \mathrm{~min}$ then $72^{\circ} \mathrm{C}$ for $10 \mathrm{~min}$ for final extension. PCR products were separated by $1.5 \%$ agarose gel electrophoresis.

Methylation-specific PCR and bisulfite sequencing analysis. Bisulfite modification of genomic DNA and methylationspecific PCR (MSP) were performed as described previously (14). Primer sequences for the DEXI promoter were 5'-ATT CGGAGGACGACGTCGCGGTC-3' and 5'-GCAAAAA CCTCGCAAAACCGACGAAA-3' for methylated reaction and 5'-TGTGATTTGGAGGATGATGTTGTGGTT-3' and 5'-CTCACAAAAACCTCACAAAACCAACAAAA-3' for unmethylated reaction. PCR started at $95^{\circ} \mathrm{C}$ for $5 \mathrm{~min}$ and $95^{\circ} \mathrm{C}$ for $45 \mathrm{sec}$ followed by 35 cycles of $95^{\circ} \mathrm{C}$ for $45 \mathrm{sec}, 53^{\circ} \mathrm{C}$ 
for $45 \mathrm{sec}$, and $72^{\circ} \mathrm{C}$ for $1 \mathrm{~min}$ then $72^{\circ} \mathrm{C}$ for $10 \mathrm{~min}$ for final extension. DNA sequencing was performed after bisulfite modification as described previously (14). Primer sequences for bisulfite sequencing were 5'-GAAGAGGGGAGGTG ATTTTG-3' and 5'-CCTACAAACCCAACACCCAT-3'. PCR started at $95^{\circ} \mathrm{C}$ for $5 \mathrm{~min}$ and $95^{\circ} \mathrm{C}$ for $45 \mathrm{sec}$ followed by 38 cycles of $95^{\circ} \mathrm{C}$ for $45 \mathrm{sec}, 56^{\circ} \mathrm{C}$ for $45 \mathrm{sec}$, and $72^{\circ} \mathrm{C}$ for $1 \mathrm{~min}$ then $72^{\circ} \mathrm{C}$ for $10 \mathrm{~min}$ for final extension. The sequences were subjected to BLAST search to identify their origins in the genome.

Real-time quantitative reverse-transcription PCR. Real-time quantitative reverse-transcription polymerase chain reaction (qRT-PCR) was carried out using LUX ${ }^{\mathrm{TM}}$ Fluorogenic Primers (Invitrogen) and SuperScript ${ }^{\mathrm{TM}}$ III Platinum ${ }^{\circledR}$ One-Step Quantitative RT-PCR System (Invitrogen) with Roche LightCycler $^{\circledR}$ (Roche Diagnostics, Mannheim, Germany) according to the manufacturers' instructions. Custom LUX ${ }^{\mathrm{TM}}$ Fluorogenic Primers (Invitrogen) were designed with D-LUX ${ }^{\mathrm{TM}}$ Designer software. Primer sequences were 5'-ACTTCACGG CGGTCGAAAGAG-3' and 5'-cgcatGATGTCTCCTGGC AAATGcG-3' where the lower case letter bases denote non-specific sequence built onto the primer to allow hairpin conformation and the bold $\mathbf{c}$ marks the position where the fluorescein FAM is attached. cDNA synthesis was performed at $45^{\circ} \mathrm{C}$ for $30 \mathrm{~min}$. PCR started at $95^{\circ} \mathrm{C}$ for $2 \mathrm{~min}$ followed by 50 cycles of $95^{\circ} \mathrm{C}$ for $5 \mathrm{sec}, 55^{\circ} \mathrm{C}$ for $10 \mathrm{sec}$, and $72^{\circ} \mathrm{C}$ for $10 \mathrm{sec}$. Expression levels of DEXI were determined by LightCycler3 Data Analysis Software, Version 3.5. Relative expression levels of DEXI are expressed as ratios (difference versus control values) between the genes of interest and an internal reference gene (GAPDH) that provides a normalization factor for the amount of RNA isolated from a specimen, and subsequently normalized with the value in the control cells.

Knockdown of DEXI by small interfering RNA. DEXI-specific small interfering RNA (siRNA) (siDEXI) was purchased from Invitrogen. The siDEXI sequence was 5'-CCUCUAUGUUC UACGUGGGCCUGUU-3'. Transfections were performed with $30 \mathrm{nM}$ of siRNA using PrimaPort siRNA Transfection Reagent ${ }^{\circledR}$ (Credia-Japan, Kyoto, Japan) in NHDF cells according to the manufacturer's instructions. BLOCK-iT ${ }^{\mathrm{TM}}$ Fluorescent Oligo (Invitrogen), fluorescein-labeled, double-stranded RNA duplex with the same length, charge, and configuration was used as control siRNA (siControl). Transfected NHDF cells were harvested for $48 \mathrm{~h}$ then used for the following experiments.

$X$-Gal cytochemical staining. X-Gal cytochemical staining for SA- $\beta$-galactosidase (SA- $\beta$-Gal) activity was performed using Senescent Cells Histochemical Staining Kit (Sigma-Aldrich, St. Louis, MO, USA) according to the manufacturer's instructions. Eight random fields were digitally photographed by phasecontrast microscope (x40 magnification) (IX51 Olympus), and SA- $\beta$-Gal-positive cells were counted by eye.

Ki-67 immunohistochemistry. Immunohistochemistry for Ki-67 was performed using anti-Ki-67 antibody (Thermo Fisher Scientific Anatomical Pathology, CA, USA) and Histofine SAB-PO ${ }^{\circledR}$ Kit (Nichirei Bioscience, Tokyo, Japan) according to the manufacturer's instructions. Positive cells were counted by eye.

Detection of apoptotic cells. Hoechst 33342 (Sigma-Aldrich) was used to observe nuclear morphologic aspects by a fluorescence microscope (Nikon BioStation IM CELL-SI-P) using a 330-380-nm filter.

Treatment with 5-aza-2'-deoxycytidine. HCT116 cells were seeded at a density of $5 \times 10^{4}$ cells on $35-\mathrm{mm}$ dishes. After incubation at $37^{\circ} \mathrm{C}$ in a humidified incubator with $5 \% \mathrm{CO}_{2}$ for $24 \mathrm{~h}$, cells were treated with $1 \mu \mathrm{M}$ of DNA demethylation agent, 5-aza-2'-deoxycytidine (DAC) for $72 \mathrm{~h}$. Then, cells were harvested for DNA and RNA extraction or used for the following experiments.

Treatment with anticancer agents CPT and 5-FU. HCT116 cells and NHDF cells were treated with CPT in $3 \mu \mathrm{M}$ final concentration for $24 \mathrm{~h}$ or with $5-\mathrm{FU}$ in $7.5 \mu \mathrm{M}$ final concentration for 5 days. Then, cells were harvested for analysis.

Transient transfection of DEXI expression plasmid. A plasmid expressing DEXI was obtained by cloning a full coding sequence of DEXI (Hokkaido System Science, Hokkaido, Japan) into the pcDNA3.1 vector (Invitrogen). A universal human reference RNA (Stratagene, Tokyo, Japan) was used to transcribe cDNA for the template DNA. PCR was performed to generate DEXI gene using this cDNA. Primer sequences were 5'-CGCGGATCCATGCTCGGCGCC-3' and 5'-CCG GAATTCCTACTCCAAGTACCCATCAAAG-3'. pcDNA3.1 plasmid DNA $(1 \mu \mathrm{g})$ containing DEXI was transfected into HCT116 cells by GeneJuice ${ }^{\circledR}$ transfection reagent (EMD Chemicals Inc., Darmstadt, Germany) according to the manufacturer's instructions followed by preparation of HCT116 cells plated at a density of $1 \times 10^{5}$ cells $/ 3.5-\mathrm{mm}$ tissue culture dish for $24 \mathrm{~h}$. Cells were assayed for DEXI expression by RT-PCR analysis then used for the following experiments.

Treatment of fluoropyrimidine and irinotecan in metastatic colorectal cancer patients and assessments. In the first-line treatment with FOLFOX, patients received every 14 days [folinic acid (FA); leucovorin] $400 \mathrm{mg} / \mathrm{m}^{2}$ infused over $2 \mathrm{~h}$ followed by 5 -FU as a $400-\mathrm{mg} / \mathrm{m}^{2}$ i.v. bolus followed by continuous 5-FU $2400 \mathrm{mg} / \mathrm{m}^{2}$ infusion over $46 \mathrm{~h}$ (LV5FU) with L-OHP $200 \mathrm{mg} / \mathrm{m}^{2}$ infusion. In the second-line treatment with FOLFIRI, patients received every 14 days the same LV5FU regimen with irinotecan $150 \mathrm{mg} / \mathrm{m}^{2}$ infusion. Sixteen patients who underwent FOLFIRI were divided into two groups according to their DEXI methylation status detected by MSP. Intensity of MSP bands was quantified by densitometry. A methylated/unmethylated (M/U)-primer ratio $>1.0$ was determined positive for DEXI methylation. Response rate (RR), disease control rate (DCR), PFS, and overall survival (OS) were assessed. Clinical features of the 16 assessed patients are shown in Table I.

Statistical analysis. Values are expressed as mean \pm SD from $\geq 3$ separate experiments. Differences of clinical data between the two patient groups were assessed by Student's t-test. Differences in response rates between the two groups were assessed 
Table I. Clinical features of 16 cases treated with FOLFIRI.

\begin{tabular}{|c|c|c|c|c|}
\hline & $\mathrm{n}$ & $\begin{array}{c}\text { Methylated } \\
(\%)\end{array}$ & $\begin{array}{c}\text { Unmethylated } \\
(\%)\end{array}$ & $\mathrm{p}$-value \\
\hline Total no. & 16 & 8 & 8 & \\
\hline Mean age & & 63.5 & 54.3 & NS \\
\hline \multicolumn{5}{|l|}{ Sex } \\
\hline Male & 12 & $8(100.0)$ & $4(50.0)$ & \multirow[t]{2}{*}{$<0.05$} \\
\hline Female & 4 & $0(0.0)$ & $4(50.0)$ & \\
\hline \multicolumn{5}{|l|}{ Primary tumor } \\
\hline Colon & 10 & $5(62.5)$ & $5(50.0)$ & \multirow[t]{2}{*}{ NS } \\
\hline Rectum & 6 & $3(37.5)$ & $3(37.5)$ & \\
\hline \multicolumn{5}{|c|}{ Metastatic organs } \\
\hline Liver & 6 & $2(25.0)$ & $4(50.0)$ & \\
\hline Lung & 3 & $2(25.0)$ & $1(12.5)$ & \\
\hline Peritoneal & 6 & $4(50.0)$ & $2(25.0)$ & \\
\hline Lymph node & 5 & $1(12.5)$ & $4(50.0)$ & \\
\hline \multicolumn{5}{|l|}{ Initial therapy } \\
\hline FOLFOX4 & 11 & $6(75.0)$ & $5(62.5)$ & \multirow[t]{2}{*}{ NS } \\
\hline FOLFOX6 & 5 & $2(25.0)$ & $3(37.5)$ & \\
\hline
\end{tabular}

by Fisher's exact test. PFS and OS were estimated by KaplanMeier method and compared between the two groups with use of the log-rank tests. Differences were considered significant at $\mathrm{p}<0.05$.

\section{Results}

DEXI frequently methylated in colorectal and gastric cancer patients. MS-AFLP with three pairs of primers yielded $\sim 170$ major bands in the fingerprints; 150 loci were assigned a number for database construction and analysis. Representative MS-AFLP fingerprints are shown in Fig. 1A. One DNA fragment, which was frequently methylated in 28 of 89 gastric cancer $(31.5 \%)$ and 33 of 73 CRC patients (49.3\%), matched the promoter sequence of DEXI. Methylated genes detected by MS-AFLP whose frequency was $>20 \%$ in CRC or gastric cancer patients are shown in Table II. MS-AFLP was validated by restriction enzyme-based methylation-specific PCR analysis. Sixty-five CRC tissues, matched normal colon epithelia, and 12 healthy normal colon epithelia were employed for this analysis. None of the normal healthy colon epithelia displayed methylation positive for DEXI. Twenty-nine of $65(45 \%)$ CRC patients exhibited methylation positive for DEXI. This frequency was consistent with that detected by MS-AFLP $(49 \%)$.

As a first step to determine whether epigenetic mechanisms are involved in silencing of DEXI gene, we performed MSP in 4 gastric cancer cell lines (H111, GSS, HGC27, AZ521), 4 CRC cell lines (CW2, CACO2, HCT116, COLO320DM) and a normal human skin fibroblast cell line (NHDF). Methylated bands were observed in 4 of 4 gastric cancer cell lines and 4 of 4 CRC cell lines. Both methylated and unmethylated bands were seen in COLO320DM. The unmethylated band is shown in NHFD (Fig. 1B). These observations suggested that gastric cancer and CRC cell lines harbored full methylation whereas COLO320DM had partial methylation and NHDF did not have DEXI methylation. These MSP findings were validated by bisulfite sequencing analysis. Degree of methylation alterations of $11 \mathrm{CpG}$ sites around transcription start site of DEXI (Fig. 1C, top) was assessed using 10 clones of each cell line and a mixture DNA of normal healthy colon epithelia. DEXI promoter was fully methylated in GSS, AZ521, HCT116 and CW2, partially methylated in COLO320DM, and unmethylated in normal tissue cell line (NHDF) as well as mixture DNA from 12 normal healthy colon epithelia (Fig. 1C, bottom). These results confirmed those detected by MSP. To verify whether aberrant methylation of DEXI was responsible for decreased levels of DEXI mRNA, relative mRNA expression levels of DEXI were quantified by qRT-PCR. We observed a marked reduction of DEXI mRNA levels in HCT116 and COLO320DM (32 and 12\%, respectively) compared with that in NHDF, where DEXI was not methylated (Fig. 1D). Relative mRNA expression levels of DEXI were in proportion to the methylation status obtained by bisulfite sequencing analysis as well as MSP analysis.

Further to estimate whether epigenetic mechanisms facilitate downregulation of DEXI expression, the epigenetic modifier DAC was applied to HCT116 and COLO320DM, where DEXI was fully or partially methylated, respectively. Levels of DEXI

Table II. Frequently methylated genes detected by MSAFLP in patients with colorectal and gastric cancer.

\begin{tabular}{|c|c|c|c|c|c|}
\hline Genes & Description & Chromosome & $\begin{array}{c}\text { Stomach }(\mathrm{n}=89) \\
\mathrm{n}(\%)\end{array}$ & $\begin{array}{l}\text { Colon }(\mathrm{n}=73) \\
\mathrm{n}(\%)\end{array}$ & $\begin{array}{c}\text { Total }(\mathrm{n}=162) \\
\mathrm{n}(\%)\end{array}$ \\
\hline DEXI & Dexamethasone-induced protein & $15 q 11.2$ & $28(31.5)$ & $36(49.3)$ & $64(39.5)$ \\
\hline ADAMTS19 & $\begin{array}{l}\text { ADAM metallopeptidase with } \\
\text { thrombospondin type } 1 \text { motif, } 19\end{array}$ & $5 q 31$ & $38(42.7)$ & $25(34.2)$ & $63(38.9)$ \\
\hline FBXL15 & F-box and leucine-rich repeat protein 15 & $10 \mathrm{q} 24.32$ & $6 \quad(6.7)$ & $34(46.6)$ & $40(24.7)$ \\
\hline PTPRN2 & $\begin{array}{l}\text { Protein tyrosine phosphatase, receptor type, } \\
\text { N polypeptide } 2\end{array}$ & $7 q 36$ & $7 \quad(7.9)$ & $33(45.2)$ & $40(24.7)$ \\
\hline NRP2 & Neuropilin 2 & $2 \mathrm{q} 33.3$ & $19(21.3)$ & $20(27.4)$ & $39(24.1)$ \\
\hline KCNMB1 & $\begin{array}{l}\text { Potassium large conductance } \\
\text { calcium-activated channel, member } 1\end{array}$ & $5 q 35.1$ & $4(4.5)$ & $30(41.1)$ & $34(21.0)$ \\
\hline
\end{tabular}




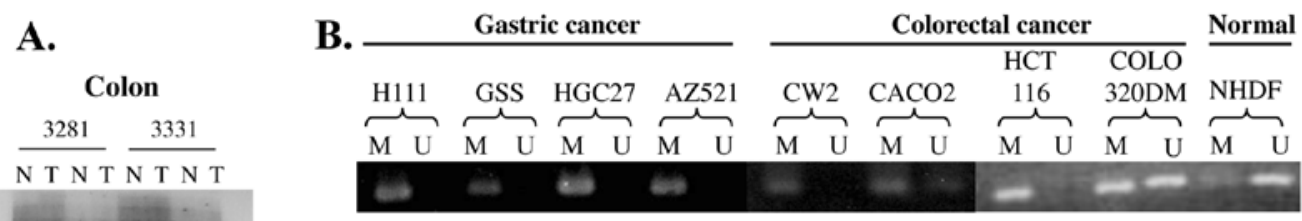

C.
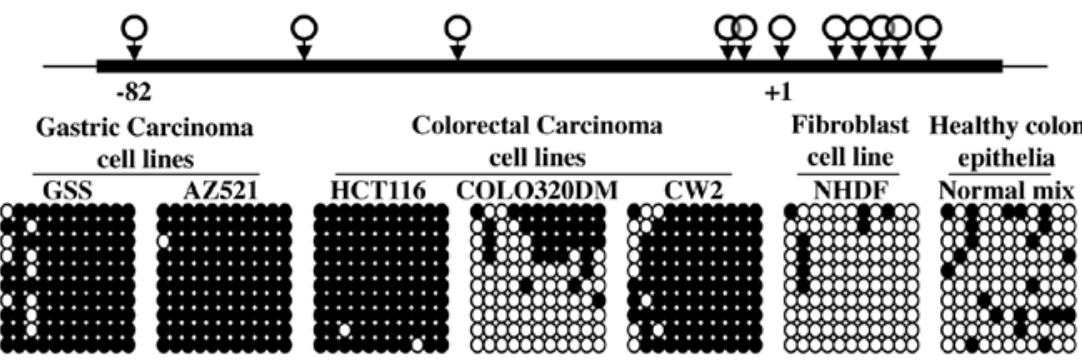

$\rightarrow *$
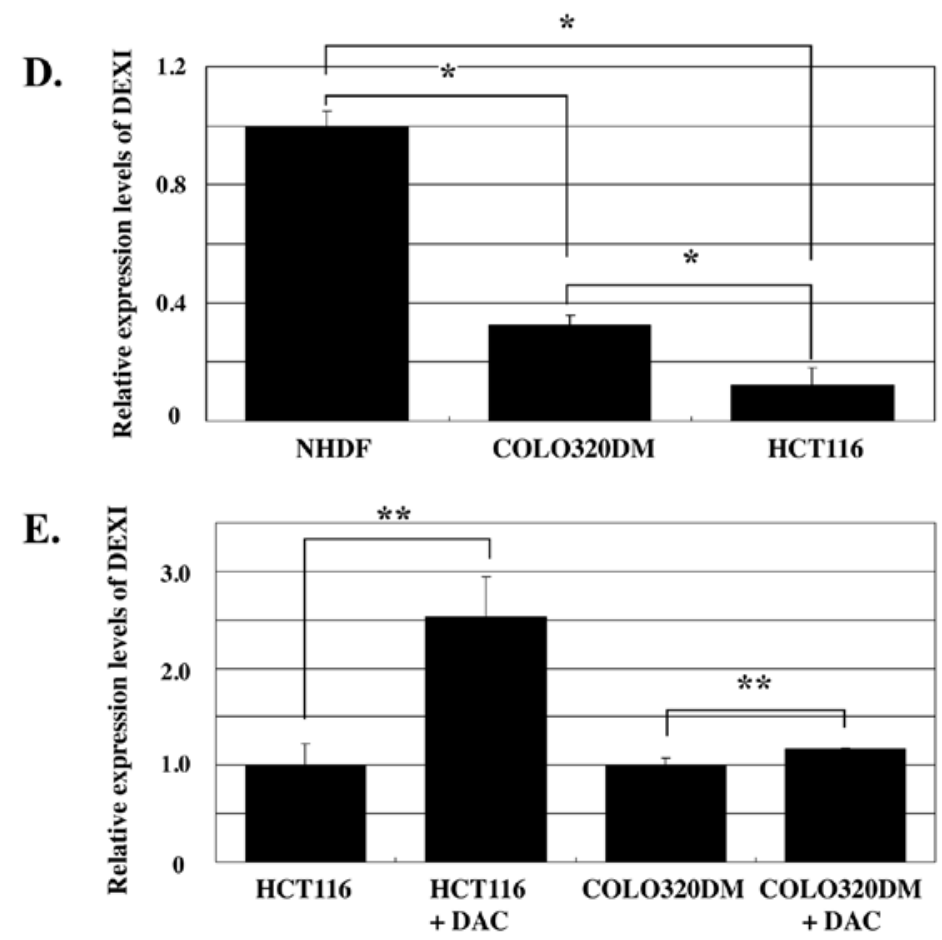

Figure 1. (A) Representative MS-AFLP fingerprints. N, corresponding normal colon epithelia; T, tumor tissue. "Hypermethylated bands displaying loss of bands in tumor assigned as DEXI; **hypomethylated bands displaying gain of bands in tumor. (B) Methylation-specific PCR (MSP) analysis of DEXI in 4 gastric cancer cell lines (H111, GSS, HGC27, AZ521), 4 colon cancer cell lines (CW2, CACO2, HCT116, Colo320DM), and a normal fibroblast cell line (NHDF). MSP products in lanes marked M show the presence of methylated templates whereas products in lanes marked $\mathrm{U}$ show the presence of unmethylated templates. (C) Schematic map of 11 CpGs around exon 1 of DEXI (top) and bisulfite sequencing analysis of DEXI (bottom). Open circles with arrows show $\mathrm{CpG}$ sites on the expanded axis. The transcription start site is marked with +1 . The amplified DEXI DNA region for sequencing analysis ranges from -402 to 48 nucleotides relative to the first exon based on DEXI cDNA sequence. The methylation status of each CpG site is indicated by closed circle (methylated) and open circle (unmethylated). (D) qRT-PCR analysis to quantify relative mRNA expression levels of DEXI. Relative values were determined by comparison with that of NHDF where DEXI was not methylated. "p<0.01. (E) Restored expression was achieved by DAC in HCT116 cells and COLO320DM cells, both of which harbored DEXI methylation. Relative expression levels were compared in cells with or without DAC treatment. ${ }^{* *} \mathrm{p}<0.05$.

mRNA in each cell line were increased depending on the degree of DEXI methylation. A 2.5-fold increase of DEXI mRNA was achieved in HCT116 where DEXI was fully methylated, whereas 1.2 -fold increase was observed in COLO320DM where DEXI was partially methylated (Fig. 1E). These results indicate that promoter hypermethylation is the predominant mechanism facilitating downregulation of DEXI gene expression in CRC cell lines.
Knockdown of mRNA level of DEXI causes resistance to CPT in NHDF cells. To investigate the biological significance of epigenetic silencing of DEXI, levels of senescence, proliferation and apoptosis were assessed following $60 \%$ knockdown of DEXI expression by siDEXI in NHDF cells. Numbers of transfected NHDF cells and levels of toxicity to NHDF cells were evaluated by BLOCK-iT Fluorescent Oligo Kit according to the manufacturer's instructions. The value of histochemical 
A.

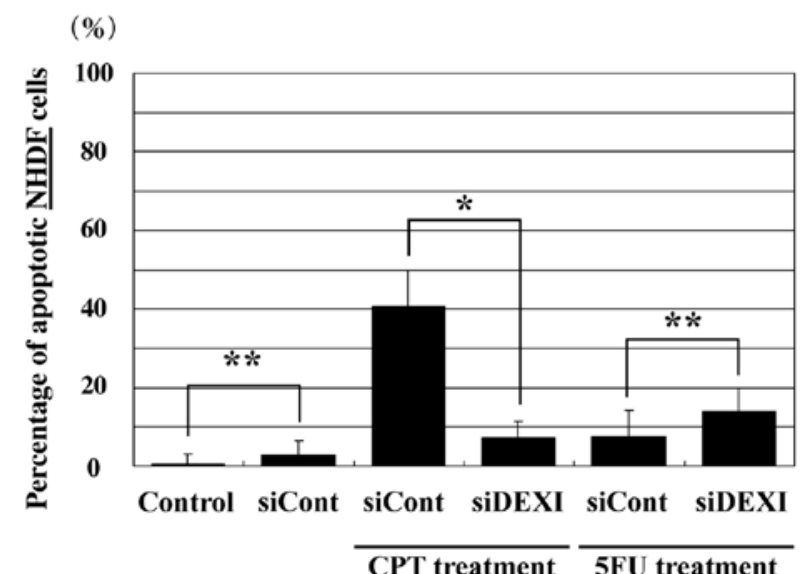

B.

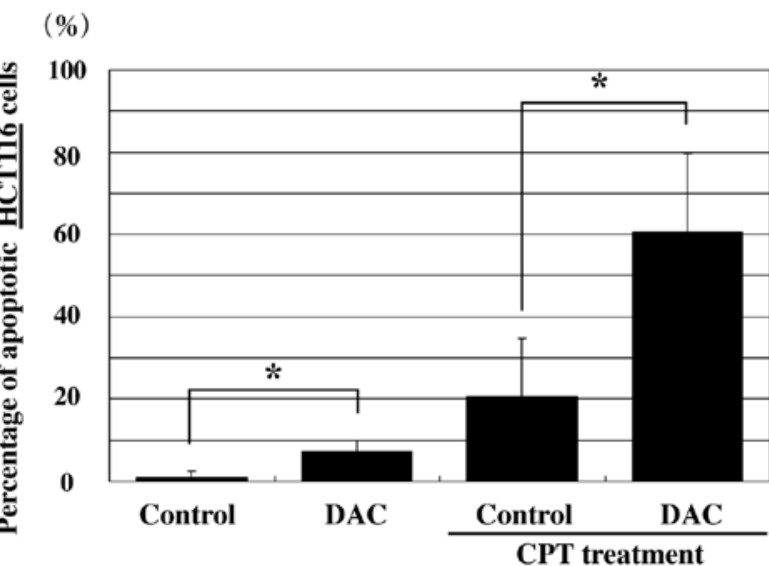

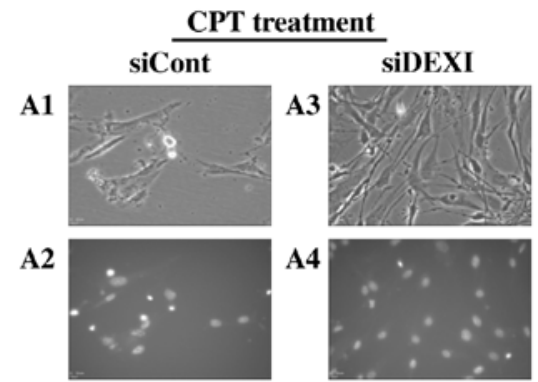

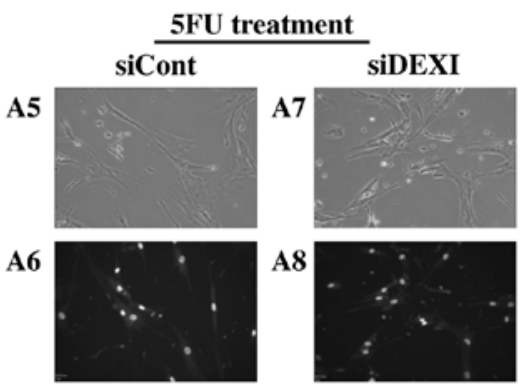

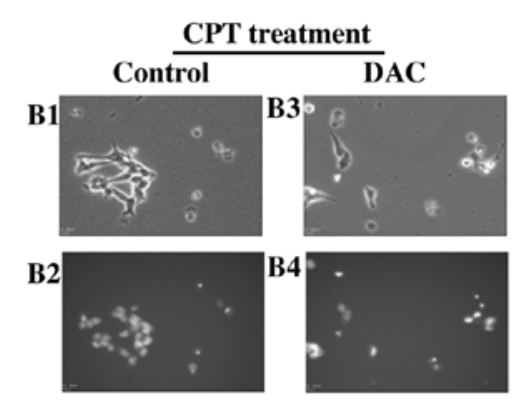

Figure 2. (A) Left panel, percent apoptotic cells observed in NHDF cells before and after transfection of siRNA (either Control-siRNA or siDEXI). Nos. apoptotic cells treated with CPT ( $24 \mathrm{~h}$ exposure) or 5-FU (5 days exposure) were compared in Control-siRNA-treated NHDF cells and siDEXI-treated NHDF cells. siCont, Control-siRNA-treated NHDF cells; siDEXI, siDEXI-treated NHDF cells. "p<0.01; * significance, NS. Right panel, appearance of cells (A1, A3, A5 and A7) and their nuclear morphologic aspects (A2, A4, A6 and A8) stained with Hoechst 33342. Representative apoptotic cells in control-siRNA-treated NHDF cells, where CPT induced 40.5\% apoptotic cells (A1 and A2) and in siDEXI-treated NHDF cells, where CPT induced 7.3\% apoptotic cells (A3 and A4). Representative apoptotic cells in control-siRNA-treated NHDF cells, where 5-FU induced 7.5\% apoptotic cells (A5 and A6) and in siDEXI-treated NHDF cells, where 5-FU induced 13.9\% apoptotic cells (A7 and A8). (B) Left panel, percent apoptotic cells observed in HCT116 cells before and after transfection of siRNA (either Control-siRNA or siDEXI). Restored expression of DEXI by treatment of DAC enhanced susceptibility of HCT116 cells harboring DEXI methylation to CPT. The number of apoptotic cells with $24 \mathrm{~h}$ exposure to CPT was compared in HCT116 cells with or without DAC treatment. "p $<0.01 ;{ }^{* *} \mathrm{p}=\mathrm{NS}$. Right panel, appearance of cells (B1 and B3) and their nuclear morphologic aspects (B2 and B4) stained with Hoechst 33342. Representative apoptotic cells in HCT116 cells without treatment of DAC, where CPT induced 20.6\% apoptotic cells (B1 and B2) and in HCT116 cells with treatment of DAC, where CPT induced $60.4 \%$ apoptotic cells (B3 and B4).

SA- $\beta$-gal staining has been demonstrated by the ability of the assay to detect oncogene-induced senescence in murine and human premalignant neoplastic lesions (15). Levels of senescence determined by SA- $\beta$-gal staining showed no significant differences of SA- $\beta$-gal staining between siDEXI-treated NHDF cells and control siRNA (siControl)-treated NHDF cells (data not shown). We next performed Ki-67 immunostaining to elucidate how DEXI participated in cell proliferation, showing no significant difference between siDEXI-treated NHDF cells and siControl-treated NHDF cells (data not shown).

We occasionally observed that apoptotic cells were decreased in siDEXI-treated NHDF cells cultured under hypoxia compared with siControl-treated NHDF cells. Therefore we applied CPT, a potent inhibitor of top I, to NHDF cells to accelerate DNA damage. CPT induced $40.5 \%$ apoptotic cells in siControltreated NHDF cells, whereas it induced $7.3 \%$ of apoptotic cells in siDEXI-treated NHDF cells ( $\mathrm{p}<0.01$; Fig. 2A). In contrast, no significant difference was observed when cells were treated with 5-FU (Fig. 2A). These results suggest that silencing of DEXI led to drug resistance to CPT in NHDF cells.

Restored DEXI expression enhances susceptibility of HCT116 to $C P T$. To elucidate the significance of DEXI gene restoration in HCT116 cells harboring DEXI methylation, cells were 
A.

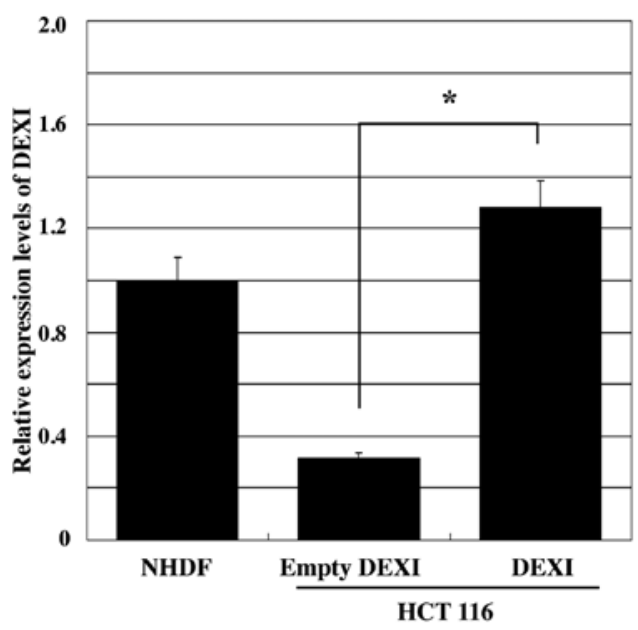

B.

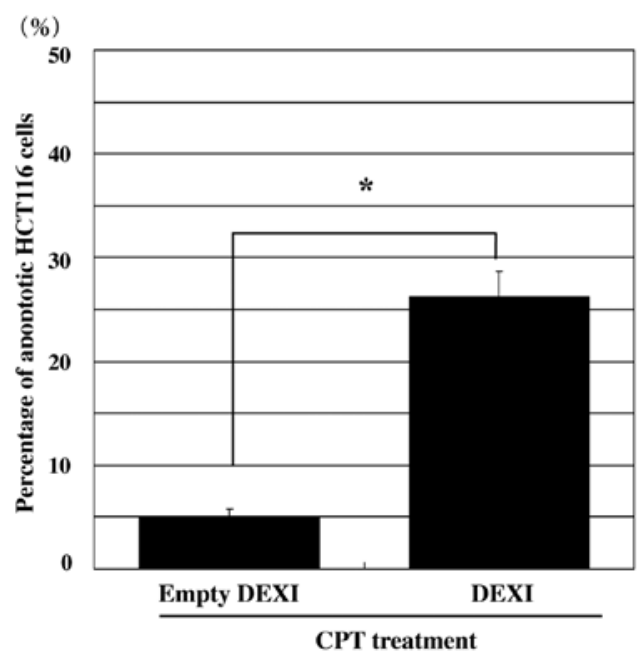

Figure 3. (A) Transient transfection of a plasmid expressing DEXI induced exogenous DEXI in HCT116 harboring DEXI methylation. Relative expression levels of DEXI were compared between HCT116 cells transfected with pcDNA3.1-control and pcDNA3.1-DEXI. Cells were analyzed $24 \mathrm{~h}$ after transfection. " p<0.01. Empty DEXI, HCT116 cells transfected with pcDNA3.1control; DEXI, HCT116 cells transfected with pcDNA3.1-DEXI. (B) Exogenous DEXI enhanced susceptibility of HCT116 cells harboring DEXI methylation to CPT. The number of apoptotic cells with $24 \mathrm{~h}$ exposure to CPT was compared between HCT116 cells transfected with pcDNA3.1-control and pcDNA3.1DEXI. ${ }^{*} \mathrm{p}<0.01$.

treated with the epigenetic modifier DAC. CPT increased apoptotic cells to $60.4 \%$ in HCT116 cells following treatment with DAC compared with $20.6 \%$ in HCT116 cells without DAC $(\mathrm{p}<0.01$; Fig. 2B). DAC achieved 3-fold increase of apoptosis in combination treatment with CPT. Since DAC has global effects on methylated genes and does not specifically target DEXI, we next determined the contribution of DEXI per se by exogenous DEXI using DEXI expression plasmid, resulting in highly efficacious induction of expression in HCT116 cells harboring DEXI methylation ( $\mathrm{p}<0.01$; Fig. 3A). Induction of apoptosis by CPT in HCT116 was $4.9 \%$ whereas significantly higher numbers of apoptotic cells $(26.4 \%$; p $<0.01$; Fig. 3B) were observed by treatment of CPT with expression plasmid of DEXI.
Methylation status of DEXI is a potent clinical biomarker for selection of patients likely to benefit from CPT-11-based chemotherapy. To estimate the clinical usefulness of DEXI methylation status as biomarker for drug resistance to CPT-11, 16 CRC patients who underwent CPT-11-based chemotherapy FOLFIRI because they were refractory to FOLFOX were analyzed for the response of the second-line chemotherapy. RR, DCR, PFS and OS were compared in two groups divided according to the methylation status of DEXI detected by MSP. Eight of 16 patients (50\%) were determined positive and eight (50\%) negative for DEXI methylation. No significant difference of clinical features was found between the two groups except no. male/female patients. RR of both groups was $0 \%$, but significant differences of DCR, PFS and OS were found between the two groups. In patients with negative DEXI methylation, DCR, PFS and OS were $62.5 \%$ and 5.3 and 15 months, respectively. On the other hand, in patients with positive DEXI methylation they were $25.0 \%$ and 2 and 11.8 months, respectively. PFS and OS are shown in Fig. 4A. To exclude the influence of first-line chemotherapy, 36 patients who underwent FOLFOX were assessed. Twenty-one of 36 patients $(58.4 \%)$ were determined positive and $15(41.6 \%)$ negative for DEXI methylation. No significant difference of RR, DCR, PFS and OS was found between the two groups in first-line treatment with FOLFOX (n=36; Fig. 4B). However, patients without DEXI methylation displayed better prognosis in the second-line treatment with FOLFIRI (Fig. 4C). These observations suggest that DEXI methylation status is a potent biomarker for selection of patients more likely to benefit from CPT-11-based chemotherapy.

\section{Discussion}

The main finding of this study is the identification of DEXI, which is frequently methylated in colorectal and gastric cancer patients. Silencing of DEXI leads to resistance to CPT but restored expression enhances susceptibility to CPT in vitro. In addition, DEXI methylation results in poor response and outcome to CPT-11-based chemotherapy, suggesting that DEXI is a potent therapeutic target and an epigenetic biomarker for the selection of patients more likely to benefit from CPT-11based chemotherapy.

Epigenetic alterations have been recognized as machinery driving cancer progression as well as carcinogenesis, which have led to a revival of interest in DNA methylation as therapeutic target in cancer (16). DAC is a nucleoside antimetabolite and potent inhibitor of DNA methyltransferase I activity, which has been approved for the treatment of myelodysplastic syndrome and other hematologic malignancies such as leukemias and lymphomas by the US Food and Drug Administration (17). However, no significant effect has been demonstrated with solid tumors $(18,19)$ probably because the DAC short half-life prevents adequate accumulation deep within solid tumors and the high doses required to achieve clinical efficacy are associated with toxicity $(20,21)$.

Recent studies succeeded in enhancing the effect of DAC in several types of solid tumors using combination therapy with anticancer agents (22-28). Some showed that DAC enhanced susceptibility to CPT $(22,23,25,29)$, which is consistent with our data. In our study, DAC achieved 3-fold increase of susceptibility 
A1.

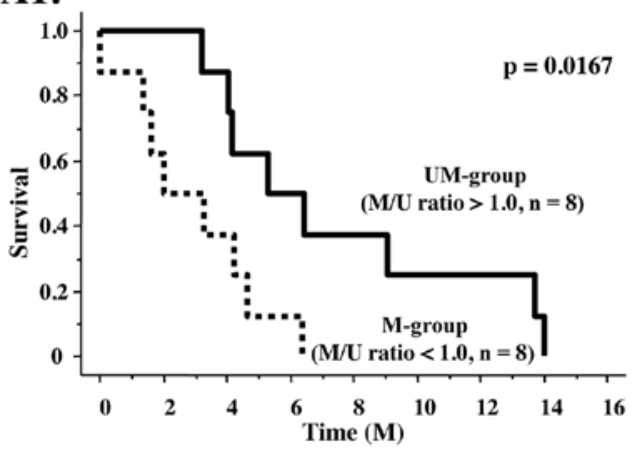

A2.

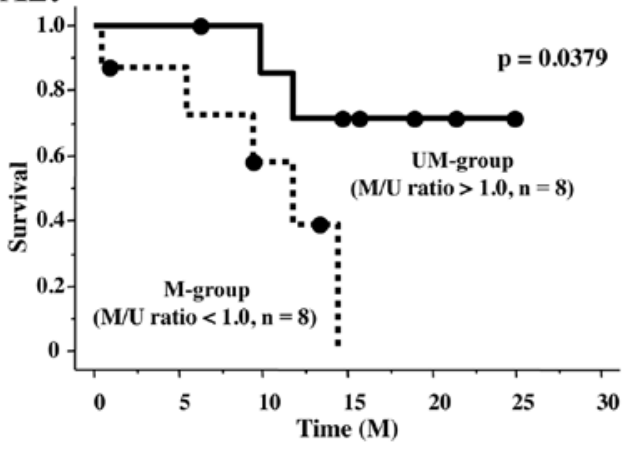

B.

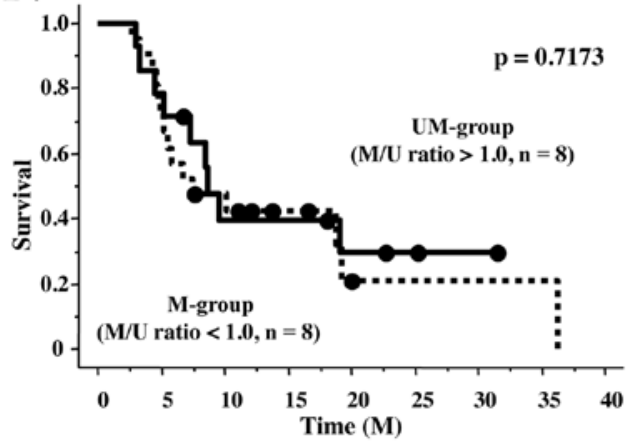

C1.

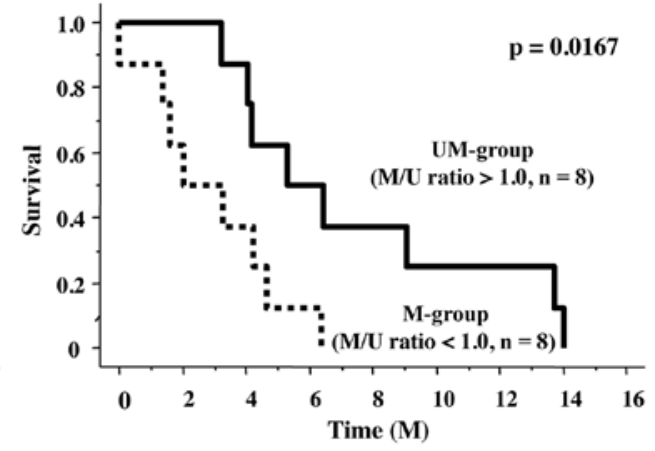

C2.

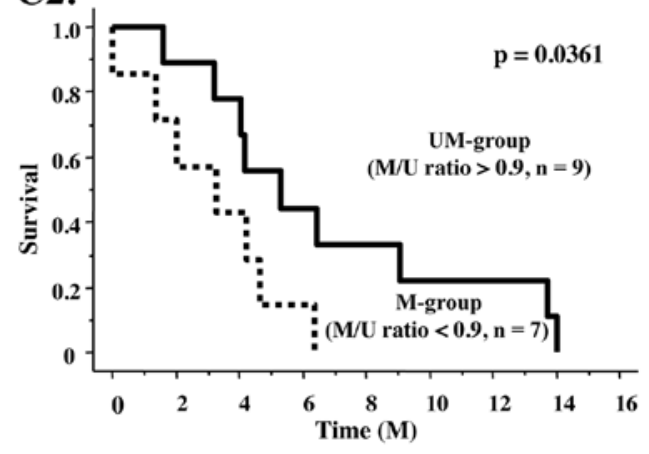

C3.

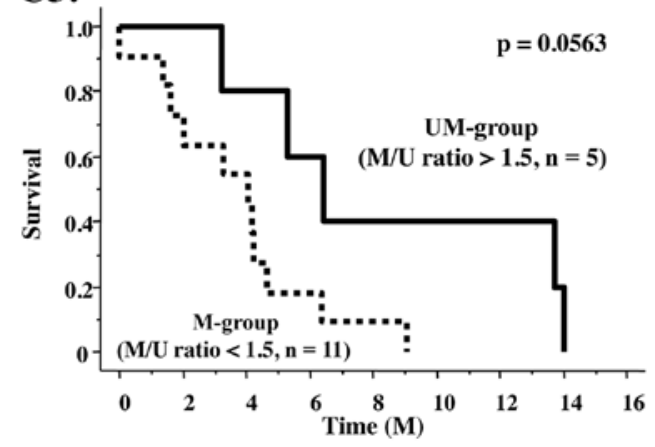

Figure 4. Survival in the two groups. UM-group (solid lines), patients without DEXI methylation; M-group (broken lines), patients with DEXI methylation. Points on the curves represent dates on which the patient data were censored. (A1) Progression-free survival (PFS) in the two groups in second-line therapy with FOLFIRI. (A2) Overall survival in the two groups in second-line therapy with FOLFIRI. (B) PFS in the two groups in first-line therapy with FOLFOX. (C) PFS in the two groups in second-line therapy with FOLFIRI according to several cut-off values.

to CPT in the colon cancer cell line, HCT116 cells harboring DEXI methylation. This increased level appears higher than that of other reports, suggesting that DAC exerted synergistic effects in combination with CPT in CRC harboring DEXI methylation. Zhang et al (28) demonstrated that no significant effects were observed in combination of DAC with 5-FU in gastric cancer cell lines, which is also consistent with our result showing no enhanced susceptibility of DAC to 5-FU in HCT116 cells. In contrast, Miza et al (25) showed DAC enhanced susceptibility of breast cancer to 5-FU. The explanation of this discrepancy is that synergistic effects of DAC in combination with CPT rather than 5-FU likely contributed to enhanced susceptibility of some types of gastrointestinal cancer harboring DEXI methylation, such as gastric cancer and CRC. Supporting this, Orta et al (26) reported that DNA demethylation protected from cleavable complex stabilization and DNA strand breakage by CPT. Thereby, DEXI methylation is likely a potent therapeutic target to facilitate optimization for cancer treatment.
Introduction of molecular targeting agents such as the antiepidermal growth factor receptor (EGFR) antibody increased survival in metastatic CRC and other cancers (30). Identification of a molecular target such as K-ras in treatment with anti-EGFR antibody enabled selection of patients more likely to benefit from chemotherapy $(31,32)$. With this knowledge, an investigation into whether DEXI could be a good predictive marker for response to CPT-based chemotherapy in CRC was undertaken. We observed better response and prognosis in FOLFOXrefractory CRC patients without DEXI methylation using CPT-11-based chemotherapy, FOLFIRI. Considering the limited response rate of $4 \%$ in second-line treatment with FOLFIRI, DEXI status could greatly contribute to selection of patients likely to benefit from CPT-11-based chemotherapy. Patients without DEXI methylation displayed better prognosis in second-line treatment with FOLFIRI, encouraging the usefulness of DEXI status as a predictive marker. Because there was no significant difference of survival in the first-line treatment 
with FOLFOX, DEXI seems not related with susceptibility to 5-FU or L-OHP. This observation regarding 5-FU is consistent with our results in vitro; no significant synergistic effects were observed by treatment with 5-FU regardless of DEXI methylation.

Recent genome project revealed a homological gene of DEXI located on chromosome 16p13.13. Then, the original DEXI located on chromosome 15 was assigned as a pseudogene although it was originally demonstrated as a real gene upregulated by dexamethasone in emphysema (12). The original DEXI comprised 95 amino acids, two of which were converted from proline to phenylalanine and alanine to glycine, respectively. It has a similar length to DEXI gene on chromosome 16 and neither stop codons nor frameshifts. Expression vector composed of sequences from the original DEXI enhanced apoptosis in HCT116 treated with CPT. Taken together, DEXI is likely transcribed and works as a real gene. However, further examinations are required to draw definitive conclusions. A possible role of decoy sequences of DEXI in chromosome 15 to cleave DEXI mRNA in chromosome 16 is feasible.

DEXI is located on chromosome 15q11.2, which is one of seven human chromosomes with a high rate of segmental duplication (33). The proximal region of $15 q$ is notable because recombination among segmental duplications can result in deletions of an imprinted gene responsible for Prader-Willi and Angelman syndromes (15q11-q13) (34). Abnormal expression of the imprinted genes results in numerous human genetic disorders including carcinogenesis (35). DEXI is not an imprinted gene; however, it may be targeted as aberrant methylation and recombination among the segmental duplications, resulting in gene inhibition. As with other heavily duplicated chromosomes, chromosome 15 has a large fraction of intrachromosomal duplication (33). MILE, a former name of DEXI, is reported to locate centromerically within flanking repeats (36). Interestingly, chromosome 16 also features one of the highest levels of segmentally duplicated sequence among human autosomes (37). Integration of these similar sequences might be induced by interchromosomal duplication between chromosome 15 and 16. Thereby, genes on chromosome 15q11-q13 such as DEXI are likely targeted as both genetic and epigenetic alterations, considering normal function of DEXI in chromosome 16 may be disrupted because of the location with a high rate of segmental duplication.

In summary, although our study results should be interpreted within the study limitations and further examinations are required to draw a definitive conclusion, we believe that our data could cast light on strategies to circumvent drug resistance by DEXI as a potent therapeutic target and an epigenetic biomarker for selection of patients most likely to benefit from CPT-11 based chemotherapy.

\section{Acknowledgments}

Our deep appreciation goes to Hiroko Yamamoto, Shinichiro Okada and Professor Toshihiro Tsuneyoshi whose comments and suggestions were of inestimable value for this study. This work was supported in part by a grant-in-aid for postgraduate students from Jichi Medical University and grant-in-aid from the Ministry of Education, Culture, Sports, Science and Technology.

\section{References}

1. de Gramont A, Figer A, Seymour M, et al: Leucovorin and fluorouracil with or without oxaliplatin as first-line treatment in advanced colorectal cancer. J Clin Oncol 18: 2938-2947, 2000.

2. Douillard JY, Cunningham D, Roth AD, et al: Irinotecan combined with fluorouracil compared with fluorouracil alone as first-line treatment for metastatic colorectal cancer: a multicentre randomised trial. Lancet 355: 1041-1047, 2000.

3. Goldberg RM, Sargent DJ, Morton RF, et al: A randomized controlled trial of fluorouracil plus leucovorin, irinotecan, and oxaliplatin combinations in patients with previously untreated metastatic colorectal cancer. J Clin Oncol 22: 23-30, 2004.

4. Saltz LB, Cox JV, Blanke C, et al: Irinotecan plus fluorouracil and leucovorin for metastatic colorectal cancer. Irinotecan Study Group. N Engl J Med 343: 905-914, 2000.

5. Liu LF, Desai SD, Li TK, Mao Y, Sun M and Sim SP: Mechanism of action of camptothecin. Ann NY Acad Sci 922: 1-10, 2000.

6. Tournigand C, Andre T, Achille E, et al: FOLFIRI followed by FOLFOX6 or the reverse sequence in advanced colorectal cancer: a randomized GERCOR study. J Clin Oncol 22: 229-237, 2004.

7. $\mathrm{Xu} \mathrm{Y}$ and Villalona-Calero MA: Irinotecan: mechanisms of tumor resistance and novel strategies for modulating its activity. Ann Oncol 13: 1841-1851, 2002.

8. Huang TT, Wuerzberger-Davis SM, Seufzer BJ, et al: NF-kappaB activation by camptothecin. A linkage between nuclear DNA damage and cytoplasmic signaling events. J Biol Chem 275: 9501-9509, 2000

9. Frigola J, Sole X, Paz MF, et al: Differential DNA hypermethylation and hypomethylation signatures in colorectal cancer. Hum Mol Genet 14: 319-326, 2005.

10. Yamamoto F, Yamamoto M, Soto JL, et al: Notl-Msell methylation-sensitive amplied fragment length polymorhism for DNA methylation analysis of human cancers. Electrophoresis 22: 1946-1956, 2001

11. Suzuki K, Suzuki I, Leodolter A, et al: Global DNA demethylation in gastrointestinal cancer is age dependent and precedes genomic damage. Cancer Cell 9: 199-207, 2006.

12. Edgar AJ, Birks EJ, Yacoub MH and Polak JM: Cloning of dexamethasone-induced transcript: a novel glucocorticoid-induced gene that is upregulated in emphysema. Am J Respir Cell Mol Biol 25: 119-124, 2001.

13. O'Shea EK, Rutkowski R and Kim PS: Evidence that the leucine zipper is a coiled coil. Science 243: 538-542, 1989.

14. Herman JG, Graff JR, Myohanen S, Nelkin BD and Baylin SB: Methylation-specific PCR: a novel PCR assay for methylation status of CpG islands. Proc Natl Acad Sci USA 93: 9821-9826, 1996.

15. Collado M and Serrano M: The power and the promise of oncogene-induced senescence markers. Nat Rev Cancer 6: 472-476, 2006.

16. Issa JP: DNA methylation as a therapeutic target in cancer. Clin Cancer Res 13: 1634-1637, 2007.

17. Kaminskas E, Farrell A, Abraham S, et al: Approval summary: azacitidine for treatment of myelodysplastic syndrome subtypes. Clin Cancer Res 11: 3604-3608, 2005.

18. Goffin J and Eisenhauer E: DNA methyltransferase inhibitorsstate of the art. Ann Oncol 13: 1699-1716, 2002.

19. Moertel CG, Schutt AJ, Reitemeier RJ and Hahn RG: Phase II study of 5-azacytidine (NSC-102816) in the treatment of advanced gastrointestinal cancer. Cancer Chemother Rep 56: 649-652, 1972.

20. Abele R, Clavel M, Dodion P, et al: The EORTC Early Clinical Trials Cooperative Group experience with 5-aza-2'-deoxycytidine (NSC 127716) in patients with colo-rectal, head and neck, renal carcinomas and malignant melanomas. Eur J Cancer Clin Oncol 23: 1921-1924, 1987.

21. Appleton K, Mackay HJ, Judson I, et al: Phase I and pharmacodynamic trial of the DNA methyltransferase inhibitor decitabine and carboplatin in solid tumors. J Clin Oncol 25: 4603-4609, 2007.

22. Ding L, Qiu L, Zhang J and Guo B: Camptothecin-induced cell proliferation inhibition and apoptosis enhanced by DNA methyltransferase inhibitor, 5-aza-2'-deoxycytidine. Biol Pharm Bull 32: 1105-1108, 2009.

23. Ishiguro $M$, Iida $S$, Uetake $H$, et al: Effect of combined therapy with low-dose 5-aza-2'-deoxycytidine and irinotecan on colon cancer cell line HCT-15. Ann Surg Oncol 14: 1752-1762, 2007. 
24. Kiziltepe T, Hideshima T, Catley L, et al: 5-Azacytidine, a DNA methyltransferase inhibitor, induces ATR-mediated DNA double-strand break responses, apoptosis, and synergistic cytotoxicity with doxorubicin and bortezomib against multiple myeloma cells. Mol Cancer Ther 6: 1718-1727, 2007.

25. Mirza S, Sharma G, Pandya P and Ralhan R: Demethylating agent 5-aza-2-deoxycytidine enhances susceptibility of breast cancer cells to anticancer agents. Mol Cell Biochem 342: 101-109, 2010.

26. Orta ML, Mateos S and Cortes F: DNA demethylation protects from cleavable complex stabilization and DNA strand breakage induced by the topoisomerase type I inhibitor camptothecin. Mutagenesis 24: 237-244, 2009.

27. Shang D, Ito N, Kamoto T and Ogawa O: Demethylating agent 5-aza-2'-deoxycytidine enhances susceptibility of renal cell carcinoma to paclitaxel. Urology 69: 1007-1012, 2007.

28. Zhang X, Yashiro M, Ohira M, Ren J and Hirakawa K: Synergic antiproliferative effect of DNA methyltransferase inhibitor in combination with anticancer drugs in gastric carcinoma. Cancer Sci 97: 938-944, 2006.

29. Crea F, Giovannetti E, Cortesi F, et al: Epigenetic mechanisms of irinotecan sensitivity in colorectal cancer cell lines. Mol Cancer Ther 8: 1964-1973, 2009.

30. Adams GP and Weiner LM: Monoclonal antibody therapy of cancer. Nature Biotechnology 23: 1147-1157, 2005.
31. Amado RG, Wolf M, Peeters M, et al: Wild-type KRAS is required for panitumumab efficacy in patients with metastatic colorectal cancer. J Clin Oncol 26: 1626-1634, 2008.

32. Lievre A, Bachet JB, Le Corre $\mathrm{D}$, et al: KRAS mutation status is predictive of response to cetuximab therapy in colorectal cancer. Cancer Res 66: 3992-3995, 2006.

33. Zody MC, Garber M, Sharpe T, et al: Analysis of the DNA sequence and duplication history of human chromosome 15. Nature 440: 671-675, 2006.

34. Christian SL, Fantes JA, Mewborn SK, Huang B and Ledbetter DH: Large genomic duplicons map to sites of instability in the Prader-Willi/Angelman syndrome chromosome region (15q11-q13). Hum Mol Genet 8: 1025-1037, 1999.

35. Falls JG,Pulford DJ, Wylie AA and Jirtle RL: Genomic imprinting: implications for human disease. Am J Pathol 154: 635-647, 1999.

36. Christian SL, Bhatt NK, Martin SA, et al: Integrated YAC contig map of the Prader-Willi/Angelman region on chromosome 15q11-q13 with average STS spacing of $35 \mathrm{~kb}$. Genome Res 8: 146-157, 1998.

37. Martin J, Han C, Gordon LA, et al: The sequence and analysis of duplication-rich human chromosome 16. Nature 432: 988-994, 2004. 